\title{
No pelvic exam, no problem: patient satisfaction following the integration of comprehensive urogynecology telemedicine
}

\author{
Stephanie Sansone $\mathrm{1}^{1,2} \mathbb{D} \cdot$ Jessica Lu ${ }^{1} \cdot$ Siri Drangsholt ${ }^{3,4} \cdot$ Tirsit S. Asfaw $^{1} \cdot$ Saya Segal ${ }^{1}$
}

Received: 11 November 2021 / Accepted: 15 January 2022 / Published online: 3 March 2022

(c) The International Urogynecological Association 2022

\begin{abstract}
Introduction and hypothesis The COVID-19 pandemic revolutionized the practice of medicine, requiring rapid adoption of telemedicine. However, patient satisfaction has not been well characterized for telemedicine visits for a broad range of urogynecologic conditions.

Methods We performed a cross-sectional survey study following a retrospective review of all urogynecologic telemedicine visits from March 1, 2020, to March 31, 2021, at a tertiary care center. The survey queried patient satisfaction using the Likert scale. Descriptive statistics and Fisher's exact analyses were performed.

Results There were 256 telemedicine visits at our institution during the study period, and 88 patients (34\% unadjusted response rate) completed the survey. The average age of study participants was 55 (SD 17; 24, 84) years old. The majority of patients were white (69\%), lived within the five boroughs of NYC (81\%), and had higher levels of education (72\% with a bachelor's or professional degree). Most visits were for urinary complaints (68\%), with those patients reporting greater fulfillment of urogynecologic needs compared to patients presenting with pelvic complaints $(p=0.02)$. There were no significant differences in satisfaction among other demographics $(p>0.05)$. Altogether, high satisfaction rates were noted for scheduling (99\%), technology (90\%), provider interaction (96\%), fulfillment of personal needs (91\%), and overall satisfaction (94\%).

Conclusions We demonstrate high patient satisfaction for telemedicine visits in a tertiary urogynecology clinic for a variety of indications, with greater fulfillment of urogynecologic needs observed for those visits which may not necessitate an inperson exam (e.g., urinary complaint).
\end{abstract}

Keywords Telemedicine and urogynecology $\cdot$ Telehealth $\cdot$ Patient satisfaction

\section{Introduction}

This work was accepted as a Scientific Salon Presentation at the American Urogynecologic Society Annual Meeting (Phoenix, Arizona; October 2021).

Stephanie Sansone

sas2064@nyp.org

1 Department of Obstetrics \& Gynecology, New YorkPresbyterian/Weill Cornell Medicine, 525 E 68th St, J130, New York, NY 10065, USA

2 Department of Urology, NewYork-Presbyterian/Weill Cornell Medicine, New York, NY 10065, USA

3 Department of Urology, New York Medical College, Valhalla, NY, USA

4 Department of Obstetrics \& Gynecology, New York Medical College, Valhalla, NY, USA
According to the Centers for Medicare and Medicaid Services, telemedicine aims to benefit patients through twoway, real-time interactive communication with a provider at a distant site [1]. The COVID-19 pandemic necessitated the rapid adoption of telemedicine to limit the spread of disease in healthcare settings. After declaration of the pandemic in March 2020, urogynecology practices at New York Presbyterian Hospital/Weill Cornell Medicine were instructed to cancel all routine visits and non-emergent surgeries. At the time, New York City (NYC) was the epicenter of the pandemic with a transmission rate five times higher than the rest of the country [2]. To prevent the spread of COVID-19, urogynecology providers replaced non-urgent office visits with telemedicine utilizing live synchronous audio and video. 
Prior to the COVID-19 pandemic, other medical fields had already started to integrate telemedicine into their practice. Not surprisingly, these specialties with early adoption were mostly within internal medicine, such as gastroenterology [3] or rheumatology [4], and primary care [5]. Yet, transitioning to telemedicine within urogynecology was not as fluid prior to the COVID-19 pandemic. Given traditional urogynecology visits typically involve a pelvic exam, urine sampling, and/or post-void residual volume, as well as other diagnostic and therapeutic procedures, patients and providers were not necessarily utilizing telemedicine. Reimbursement is also typically less for telemedicine visits, which could have been an additional deterrent. The first publication regarding the use of mobile technology in urogynecology did report that patients were willing to use telemedicine [6]. Additionally, randomized controlled trials have since evaluated postoperative urogynecology visits, by both telephone [7] and video [8], compared to traditional in-office visits, and both investigators found telemedicine visits non-inferior to standard office visits in terms of satisfaction and patient outcomes. However, it is unknown whether telemedicine can be applied to a broader range of urogynecologic conditions without compromising patient satisfaction.

COVID-19 accelerated the specialty's transition to telemedicine, with expert consensus guidelines being developed for the evaluation and management of a broad spectrum of urogynecologic conditions [9]. Given our institution's rapid integration of telemedicine within the nation's first epicenter, and continued usage even after the acute pandemic, we sought to determine whether women with urogynecologic conditions are adequately accessed and satisfied using this modality. With telemedicine challenging traditional methods of care as the "new normal," this study aims to evaluate patient satisfaction and fulfillment of urogynecologic needs using telemedicine across a comprehensive range of chief complaints and diagnoses of pelvic floor disorders.

\section{Materials and methods}

We performed a cross-sectional survey study following a retrospective review of all urogynecologic telemedicine visits in the Department of Obstetrics and Gynecology from March 1, 2020, to March 31, 2021, at New York Presbyterian Hospital/ Weill Cornell Medicine. Institutional Review Board approval was obtained for this study. All patients who contacted the department to make a urogynecology appointment, regardless of chief complaint or whether they were establishing care as a new patient or returning for a follow-up visit, were offered a telemedicine visit. Those who accepted and completed the telemedicine visit were sent the survey and included in the study. Those patients who were scheduled for a telemedicine visit and did not complete the visit (i.e., "no-show") were excluded. Patients who were non-English speaking or unable to complete the written survey were excluded as well. Live, two-way synchronous video visits were performed unless connectivity issues arose that necessitated a phone visit. Video visits were performed using the videoconferencing platform Zoom, which was initiated through the electronic medical record (Epic), on a mobile device or computer with video capabilities. All patients were required to have an email address on file to use the electronic medical record platform for the telemedicine visit.
Fig. 1 Patient urogynecologic telemedicine survey. A depiction of our 19-question survey instrument using the Likert scale

\begin{tabular}{|c|c|c|c|c|c|}
\hline & $\begin{array}{l}\text { Strongly } \\
\text { Disagree }\end{array}$ & Disagree & $\begin{array}{l}\text { Neither Agree } \\
\text { nor Disagree }\end{array}$ & Agree & $\begin{array}{l}\text { Strongly } \\
\text { Agree }\end{array}$ \\
\hline \multicolumn{6}{|l|}{ Scheduling } \\
\hline \multicolumn{6}{|l|}{ I was able to schedule my telehealth visit easily. } \\
\hline \multicolumn{6}{|l|}{ My telehealth visit began on time. } \\
\hline \multicolumn{6}{|l|}{ There were convenient visit times and dates to speak with my doctor. } \\
\hline \multicolumn{6}{|l|}{ Technology } \\
\hline \multicolumn{6}{|l|}{ I had no difficulty connecting for telehealth visits. } \\
\hline \multicolumn{6}{|l|}{ The connection was of good quality for the telehealth visits. } \\
\hline \multicolumn{6}{|l|}{ Provider } \\
\hline \multicolumn{6}{|l|}{ My doctor introduced herself and her role in my care. } \\
\hline \multicolumn{6}{|l|}{ My doctor was courteous. } \\
\hline \multicolumn{6}{|l|}{ My doctor was skillful and knowledgeable. } \\
\hline \multirow{2}{*}{\multicolumn{6}{|c|}{$\begin{array}{l}\text { My doctor took time to listen to me. } \\
\text { My prescriptions and orders were placed without delay. }\end{array}$}} \\
\hline & & & & & \\
\hline \multirow{2}{*}{\multicolumn{6}{|c|}{$\begin{array}{l}\text { My questions and concerns were addressed during the visit. } \\
\text { Personal Needs }\end{array}$}} \\
\hline & & & & & \\
\hline \multicolumn{6}{|l|}{ I do not have a concern for my privacy. } \\
\hline \multicolumn{6}{|l|}{ The visit fulfilled my urogynecologic needs. } \\
\hline \multicolumn{6}{|l|}{ My concerns were addressed during the visit. } \\
\hline \multirow{2}{*}{\multicolumn{6}{|c|}{$\begin{array}{l}\text { It was easy to access the telehealth doctor. } \\
\text { Overall Satisfaction }\end{array}$}} \\
\hline & & & & & \\
\hline \multicolumn{6}{|l|}{$\begin{array}{l}\text { Overall Satisfaction } \\
\text { I was happy with the visit. }\end{array}$} \\
\hline \multicolumn{6}{|l|}{ I was satisfied with the telehealth urogynecologic care. } \\
\hline \multicolumn{6}{|l|}{ I am likely to recommend my telehealth urogynecologist doctor. } \\
\hline I am likely to continue to seek care at the urogynecology $d$ & & & & & \\
\hline
\end{tabular}


Patients were then contacted by email to complete a 19-question survey (Fig. 1), and patients' demographics and their reason for visit were extracted from the electronic medical record. The patients were emailed the survey and electronic consent form twice, and for those who did not respond, phone interviews and consent were obtained. Timing of survey distribution during the study period varied given the need for Institutional Review Board (IRB) approval and pandemic-related IRB delays. However, in

Table 1 Patient demographics and visit type, $n=88$

\begin{tabular}{|c|c|}
\hline & $n(\%$ of total $)$ \\
\hline \multicolumn{2}{|l|}{ Age } \\
\hline 20-39 years & $19(22 \%)$ \\
\hline $40-59$ years & $32(36 \%)$ \\
\hline $60-79$ years & $32(36 \%)$ \\
\hline $80+$ years & $5(6 \%)$ \\
\hline \multicolumn{2}{|l|}{ Race, ethnicity } \\
\hline White & $61(69 \%)$ \\
\hline Hispanic & $14(16 \%)$ \\
\hline Asian & $7(8 \%)$ \\
\hline Black & $4(5 \%)$ \\
\hline Other & $2(2 \%)$ \\
\hline \multicolumn{2}{|l|}{ Location } \\
\hline Manhattan* & $34(39 \%)$ \\
\hline Queens* & $16(18 \%)$ \\
\hline Brooklyn* & $11(13 \%)$ \\
\hline Bronx* & $9(10 \%)$ \\
\hline Staten Island* & $1(1 \%)$ \\
\hline Long Island & $4(5 \%)$ \\
\hline New York State & $5(6 \%)$ \\
\hline New Jersey & $6(7 \%)$ \\
\hline Connecticut & $2(2 \%)$ \\
\hline \multicolumn{2}{|l|}{ Education } \\
\hline Less than high school & $2(2 \%)$ \\
\hline High school or equivalent & $15(17 \%)$ \\
\hline Some college (no degree) & $1(1 \%)$ \\
\hline Bachelor's degree & $42(48 \%)$ \\
\hline Professional degree & $21(24 \%)$ \\
\hline Did not answer & $7(8 \%)$ \\
\hline \multicolumn{2}{|l|}{ Visit type } \\
\hline Urinary complaint & $60(68 \%)$ \\
\hline Urinary tract infection (UTI) & $27(31 \%)$ \\
\hline Incontinence & $18(20 \%)$ \\
\hline Lower urinary tract symptoms (LUTS) & $15(17 \%)$ \\
\hline Pelvic complaint & $28(32 \%)$ \\
\hline Prolapse & $24(27 \%)$ \\
\hline Vulvovaginal symptoms & $4(5 \%)$ \\
\hline
\end{tabular}

*Indicates one of the boroughs of New York City, NYC general, the survey was emailed out twice to patients within a 30-day period. If there was no response following the second email, a telephone call attempt was made during the following 30 days. Patients were then considered a nonresponse if they did not respond to the phone call. An unadjusted response rate was calculated for those patients who were sent the survey.

Our survey was adopted from an existing multi-domain questionnaire that had been used to assess virtual obstetric care satisfaction [10]. The survey had not been previously tested in our study population, but the instrument included a comprehensive collection of categories related to telemedicine satisfaction in a straightforward format. Specifically, our survey included five domains-scheduling (3 questions), technology (2 questions), provider interaction (6 questions), fulfillment of personal needs (4 questions), and overall satisfaction (4 questions). Responses were recorded using the Likert scale and ultimately grouped as either "satisfied" ("strongly agree" and "agree") versus "dissatisfied" ("strongly disagree," "disagree," and "neither agree or disagree"). Patient demographics, including age, race, location, and education level, and visit types, categorized as either a urinary or pelvic complaint, were also grouped for analysis.

Descriptive statistics were performed for demographic variables. Two questions were selected for further analysis to represent overall patient fulfillment and satisfaction, including "The visit fulfilled my urogynecologic needs" and "I was satisfied with the telehealth urogynecologic care." Fisher's exact analyses were performed to assess for differences in the responses to these two questions among demographic groups and by visit type. GraphPad Prism version 9.2.0 was used to perform Fisher's exact analyses. A $p$ value $<0.05$ was considered statistically significant.

\section{Results}

There were 256 telemedicine visits completed at our institution during the study period, and 88 patients completed the survey (34\% unadjusted response rate). More visits were conducted by video $(n=77,87.5 \%)$ than by phone $(n=11$, $12.5 \%)$, and 57 patients (65\%) had prior experience with telemedicine. An additional 34 telemedicine visits had been scheduled, but these patients did not connect to the medical record virtual platform or answer a phone call at their appointment time, leading to a "no-show" rate of $12 \%$ $(n=34 / 290)$. These 34 patients were not included in our analysis.

Descriptive statistics for demographic variables are shown in Table 1 . The average age of participants was 55 
Table 2 Survey responses

Satisfied $n(\% \quad$ Dissatisfied of row total) $n$ (\% of row total)

\begin{tabular}{|c|c|c|}
\hline \multicolumn{3}{|l|}{ Scheduling } \\
\hline I was able to schedule my telehealth visit easily & $88(100 \%)$ & $0(0 \%)$ \\
\hline My telehealth visit began on time & $85(97 \%)$ & $3(3 \%)$ \\
\hline There were convenient visit times and dates to speak with my doctor & $88(100 \%)$ & $0(0 \%)$ \\
\hline Composite, all scheduling questions & $261(99 \%)$ & $3(1 \%)$ \\
\hline \multicolumn{3}{|l|}{ Technology } \\
\hline I had no difficulty connecting for telehealth visits & $77(87.5 \%)$ & $11(12.5 \%)$ \\
\hline The connection was of good quality for the telehealth visits & $81(92 \%)$ & $7(8 \%)$ \\
\hline Composite, all technology questions & $158(90 \%)$ & $18(10 \%)$ \\
\hline \multicolumn{3}{|l|}{ Provider interaction } \\
\hline My doctor introduced herself and her role in my care & $86(98 \%)$ & $2(2 \%)$ \\
\hline My doctor was courteous & $87(99 \%)$ & $1(1 \%)$ \\
\hline My doctor was skillful and knowledgeable & $88(100 \%)$ & $0(0 \%)$ \\
\hline My doctor took time to listen to me & $86(98 \%)$ & $2(2 \%)$ \\
\hline My prescriptions and orders were placed without delay & $81(92 \%)$ & $7(8 \%)$ \\
\hline My questions and concerns were addressed during the visit & $77(87.5 \%)$ & $11(12.5 \%)$ \\
\hline Composite, all provider questions & $505(96 \%)$ & $23(4 \%)$ \\
\hline \multicolumn{3}{|l|}{ Fulfillment of personal needs } \\
\hline I do not have a concern for my privacy & $87(99 \%)$ & $1(1 \%)$ \\
\hline The visit fulfilled my urogynecologic needs & $68(77 \%)$ & $20(23 \%)$ \\
\hline My concerns were addressed during the visit & $80(91 \%)$ & $8(9 \%)$ \\
\hline It was easy to access the telehealth doctor & $86(98 \%)$ & $2(2 \%)$ \\
\hline Composite, all personal questions & $321(91 \%)$ & $31(9 \%)$ \\
\hline \multicolumn{3}{|l|}{ Overall satisfaction } \\
\hline I was happy with the visit & $82(93 \%)$ & $6(7 \%)$ \\
\hline I was satisfied with the telehealth urogynecologic care & $79(90 \%)$ & $9(10 \%)$ \\
\hline I am likely to recommend my telehealth urogynecologist doctor & $83(94 \%)$ & $5(6 \%)$ \\
\hline I am likely to continue to seek care at the urogynecology department & $87(99 \%)$ & $1(1 \%)$ \\
\hline Composite, all overall satisfaction questions & $331(94 \%)$ & $21(6 \%)$ \\
\hline
\end{tabular}

Responses were classified as satisfied if the patient answered "strongly agree" or "agree," while responses were classified as dissatisfied if they answered "strongly disagree," "disagree," or "neither agree or disagree"

( $\mathrm{SD}=17$, range $=24-84)$ years old. The majority of patients were white $(69 \%)$, lived within the five boroughs of NYC $(81 \%)$, and had higher levels of education (72\% with a bachelor's or professional degree). Visit types are also categorized by urinary or pelvic complaints in Table 1. Urinary complaints comprised $68 \%$ of visits, and the remainder were for pelvic complaints (32\%). Survey responses for each question are shown in Table 2. Composite responses for each subcategory were also calculated, with high composite satisfaction scores noted for each category.

Overall, high satisfaction was noted across all demographic groups (Tables 3 and 4). There were no significant differences in satisfaction noted among age, race, location, and education. Greater fulfillment of urogynecologic needs was noted for those patients with urinary complaints compared to pelvic complaints (Table 3; $p=0.02$ ), but there was no significant difference in overall satisfaction between these two groups (Table $4 ; p=0.14$ ).

\section{Discussion}

Our survey study found that urogynecologic patients exhibit high satisfaction with the integration of telemedicine across an array of chief complaints and diagnoses. Regardless of the healthcare specialty, the COVID-19 pandemic required rapid adoption of telemedicine. Urogynecology is no exception, with the added significance of being able to protect a potentially more vulnerable patient population while still establishing meaningful patient-physician relationships. 
Table 3 Associations of patient demographics and visit type with fulfillment of urogynecologic needs

$\begin{array}{lll}\text { Satisfied } n(\% & \text { Dissatisfied } & p \text { value } \\ \text { of row total }) & n(\% \text { of row } \\ & \text { total })\end{array}$

Age

20-59 years

$60+$ years

$42(82 \%)$

$9(18 \%)$

$11(30 \%)$

Race

White

$48(79 \%)$

$13(21 \%)$

Other

$20(74 \%)$

$7(26 \%)$

Location

5 boroughs of NYC

$52(73 \%)$

$19(27 \%)$

Outside of NYC

$16(94 \%)$

$1(6 \%)$

Education*

Less than college

$11(61 \%)$

$7(39 \%)$

College degree or more

$51(81 \%)$

Visit type

Urinary complaint

UTI

$51(85 \%)$

$9(15 \%)$

Incontinence

LUTS

Pelvic complaint

Prolapse

$17(61 \%)$

Vulvovaginal

Responses were classified as satisfied if the patient answered "strongly agree" or "agree" to the statement, "The visit fulfilled my urogynecologic needs," while responses were classified as dissatisfied if they answered "strongly disagree," "disagree," or "neither agree or disagree." $P$ value refers to the Fisher's exact test, and those $p<0.05$ are considered significant

*Those who did not state their education level $(n=7)$ were excluded

Expedited guidelines for the delivery of urogynecologic care were established for providers [9], and prior studies show that this patient population is willing to employ telemedicine [6]. Yet, understanding patient satisfaction with this modality is crucial to know whether providers should continue using telemedicine for any urogynecologic indication. High telemedicine satisfaction has been noted in the field of urology in an urban NYC patient population [11], but whether this can be applied to urogynecology is important to decipher. Our survey-based study, which took place at a NYC tertiary academic medical center in the first US epicenter of the pandemic, provides helpful insights into the value of telemedicine for urogynecology visits.

We found that patient satisfaction was high in all demographic groups and across all subcategories of our survey instrument. Patients in close proximity to the Manhattan medical center ( $81 \%$ lived in NYC) and with higher education levels (72\% with bachelor's degree or higher) trended towards higher utilization of telemedicine. This contrasts another study, which was completed at an academic medical center in the Bronx, where video visits were declined by $75 \%$ of their patients, with $50 \%$ of those attributing that decision to lack of electronic device or internet signal [12]. We found there were no significant differences in satisfaction between age, race, location, and education $(p>0.05)$. However, compared to those patients who lived within the five boroughs of NYC, those patients outside of NYC trended towards greater fulfillment of urogynecologic needs ( $94 \%$ vs. $73 \% ; p=0.11)$ and higher overall satisfaction $(100 \%$ vs. $87 \% ; p=0.20$ ). This trend may be due to increased access to tertiary care via telemedicine for those who live far away from the urban medical center. A trend towards greater satisfaction was also observed for those with higher levels of education. Those patients with a college degree or more reported greater fulfillment of urogynecologic needs $(81 \%$ vs. $61 \% ; p=0.11)$. While not statistically significant, this perhaps may be due to those with higher levels of education having greater health literacy and understanding of explanations of pelvic floor disorders via telemedicine. This could be evaluated further with a greater sample size and more diverse population to assess whether higher levels of education lead to greater comprehension following telemedicine visits.

Importantly, we did note significant differences in satisfaction by visit type, defined as visits for pelvic versus urinary complaints. Patients had greater fulfillment of urogynecologic needs for urinary complaints compared to pelvic complaints ( $85 \%$ vs. $61 \%$; $p=0.02)$. Given that visits for urinary complaints (i.e., overactive bladder, incontinence) do not necessarily warrant an urgent in-person exam, this may lead to greater satisfaction. Providers may be able to implement telemedicine visits for urinary complaints and then schedule more urgent appointments necessitating an exam in the office. This could expedite diagnosis and treatment for those patients with prolapse, vaginal cysts, or vulvovaginal disease with more in-office capacity. For those patients with urinary complaints, in which the first line of therapy is often behavior modifications and conservative measures, care may be initiated sooner with counseling conducted via telemedicine. Finally, despite this difference in fulfillment of needs between urinary and pelvic complaints, there was not a significant difference in overall satisfaction between visit types ( $93 \%$ vs. $82 \%$; $p=0.14)$. Therefore, providers should feel reassured that patients are still satisfied with their telemedicine visit regardless of the reason for their visit.

Our study has several limitations, including a low response rate. While there are standardized survey response rate calculators that take into account additional variables (i.e., confirmation that the patient received the emailed survey), we were unable to account for these metrics and thus could not calculate an adjusted response rate. For example, while the patient may have had an active email account 
Table 4 Associations of patient demographics and visit type with overall satisfaction

\begin{tabular}{|c|c|c|c|}
\hline & $\begin{array}{l}\text { Satisfied } \\
n(\% \text { of row total) }\end{array}$ & $\begin{array}{l}\text { Dissatisfied } \\
n \text { (\% of row total) }\end{array}$ & $p$ value \\
\hline \multicolumn{4}{|l|}{ Age } \\
\hline $20-59$ years & $48(94 \%)$ & $3(6 \%)$ & \multirow[t]{2}{*}{0.16} \\
\hline $60+$ years & $31(84 \%)$ & $6(16 \%)$ & \\
\hline \multicolumn{4}{|l|}{ Race } \\
\hline White & $54(89 \%)$ & $7(11 \%)$ & \multirow[t]{2}{*}{0.72} \\
\hline Other & $25(93 \%)$ & $2(7 \%)$ & \\
\hline \multicolumn{4}{|l|}{ Location } \\
\hline 5 boroughs of NYC & $62(87 \%)$ & $9(13 \%)$ & \multirow[t]{2}{*}{0.20} \\
\hline Outside of NYC & $17(100 \%)$ & $0(0 \%)$ & \\
\hline \multicolumn{4}{|l|}{ Education* } \\
\hline Less than college degree & $15(83 \%)$ & $3(17 \%)$ & \multirow[t]{2}{*}{0.41} \\
\hline College degree or more & $57(90 \%)$ & $6(10 \%)$ & \\
\hline \multicolumn{4}{|l|}{ Visit type } \\
\hline \multicolumn{3}{|l|}{ Urinary complaint } & \multirow[t]{4}{*}{0.14} \\
\hline UTI & $56(93 \%)$ & $4(7 \%)$ & \\
\hline \multirow{2}{*}{\multicolumn{3}{|c|}{$\begin{array}{l}\text { Incontinence } \\
\text { LUTS }\end{array}$}} & \\
\hline & & & \\
\hline \multicolumn{4}{|l|}{ Pelvic complaint } \\
\hline Prolapse & $23(82 \%)$ & $5(18 \%)$ & \\
\hline Vulvovaginal & & & \\
\hline
\end{tabular}

Responses were classified as satisfied if the patient answered "strongly agree" or "agree" to the statement "I was satisfied with the telehealth urogynecologic care," while responses were classified as dissatisfied if they answered "strongly disagree," "disagree," or "neither agree or disagree." $P$ value refers to the Fisher's exact test, and those $p<0.05$ are considered significant

*Those who did not state their education level $(n=7)$ were excluded associated with the electronic medical record system at the time of the telemedicine visit, we do not know whether this account was still active and/or appropriately linked when the survey was distributed. As with any survey study, there is also the potential for bias due to voluntary participation, which both affects response rate and possibly skews the responses. Dissatisfaction with the visit could very well have caused patients not to complete the survey, which may lower our response rate and limit our responders to only those with high satisfaction. This bias may be amplified in our survey about telemedicine given the behavioral overlap. Individuals dissatisfied with telemedicine may also have an aversion to phone calls and surveys. Additionally, we did not capture data from those patients who "no-showed" their visits, which could have impacted the survey responses. Another source of bias may stem from the high education level of the majority of patients. In an effort to limit these biases, additional metrics could be tracked to more accurately calculate an adjusted response rate. The survey instrument was also not standardized or validated for a urogynecologic population, and recall bias is another factor to take into account when considering the timing of patients' survey completion. Finally, without the pressure of the pandemic, these results may not be applicable at another timepoint. A future study could assess whether telemedicine remains highly satisfying to patients without the acute concerns of the pandemic limiting in-person urogynecologic visits. This future study could be helpful in validating the questionnaire as well.

A strength of this study is that we assessed satisfaction across all chief complaints/visit types during the pandemic. Given this study was conducted in the first US epicenter of the COVID-19 pandemic, telemedicine was promptly adopted in March 2020 with the immediate need to limit in-person visits and reallocate healthcare resources in NYC. Thus, these data are helpful in reinforcing that telemedicine is not only feasible, but also fulfilling, for patients even during the strictest of pandemic lockdowns. Even though our clinic has opened up for more in-person visits compared to the start of the pandemic, our institution has continued to conduct telemedicine visits with positive reviews. This demonstrates that this model is sustainable.

Overall, our study demonstrates high patient satisfaction across all demographics and visit types for telemedicine visits in a tertiary urogynecology clinic during the COVID-19 pandemic. Greater fulfillment of urogynecologic needs was observed for those visits for urinary complaints, which may not necessitate an in-person exam. Future studies are warranted to standardize and validate a telemedicine satisfaction 
survey for urogynecology and to determine which patients may benefit the most from this technology.

Acknowledgments The authors would like to acknowledge Dr. Paul Christos in the Division of Biostatistics and Department of Population Health Sciences at Weill Cornell Medicine for his assistance with the statistical analyses.

Author participation S Sansone: protocol/project development, data collection, data analysis, manuscript writing.

$\mathrm{J} \mathrm{Lu}$ : protocol/project development, data collection, manuscript editing.

S Drangsholt: data collection, data analysis, manuscript editing.

TS Asfaw: data collection, manuscript editing.

S Segal: protocol/project development, data collection, data analysis, manuscript writing.

\section{Declarations}

\section{Conflict of interest None.}

\section{References}

1. Medicaid.gov. Telemedicine I Medicaid. 2021 [online] Available at: https://www.medicaid.gov/medicaid/benefits/telemedicine/ index.html. Accessed 29 Sept 2021.

2. Stier AJ, Berman MG, Bettencourt LMA. COVID-19 attack rate increases with city size (March 30, 2020). Mansueto Inst Urban Innov Res Pap No. 19. 2020. https://ssrn.com/abstract=3564464.

3. Helsel BC, Williams JE, Lawson K, Liang J, Markowitz J. Telemedicine and Mobile health technology are effective in the management of digestive diseases: a systematic review. Dig Dis Sci. 2018;63(6):1392-408. https://doi.org/10.1007/ s10620-018-5054-z.

4. Kataria S, Ravindran V. Digital health: a new dimension in rheumatology patient care. Rheumatol Int. 2018;38(11):1949-57. https://doi.org/10.1007/s00296-018-4037-x.
5. Bashshur RL, Howell JD, Krupinski EA, Harms KM, Bashshur N, Doarn CR. The empirical foundations of telemedicine interventions in primary care. Telemed J E Health. 2016;22(5):342-75. https://doi.org/10.1089/tmj.2016.0045.

6. Lee DD, Arya LA, Andy UU, Sammel MD, Harvie HS. Willingness of women with pelvic floor disorders to use mobile technology to communicate with their health care providers. Female Pelvic Med Reconstr Surg. 2019;25(2):134-8. https://doi.org/10. 1097/SPV.0000000000000668.

7. Thompson JC, Cichowski SB, Rogers RG, et al. Outpatient visits versus telephone interviews for postoperative care: a randomized controlled trial. Int Urogynecol J. 2019;30(10):1639-46. https:// doi.org/10.1007/s00192-019-03895-z.

8. Lee DD, Arya LA, Andy UU, Harvie HS. Video virtual clinical encounters versus office visits for postoperative care after pelvic organ prolapse surgery: a randomized clinical trial. Female Pelvic Med Reconstr Surg. 2021;27(7):432-8. https://doi.org/10.1097/ SPV.0000000000000909.

9. Grimes CL, Balk EM, Crisp CC, et al. A guide for urogynecologic patient care utilizing telemedicine during the COVID-19 pandemic: review of existing evidence. Int Urogynecol J. 2020;31(6):1063-89. https://doi.org/10.1007/ s00192-020-04314-4.

10. Pflugeisen BM, Mou J. Patient satisfaction with virtual obstetric care. Matern Child Health J. 2017;21(7):1544-51. https://doi.org/ 10.1007/s10995-017-2284-1.

11. Allen AZ, Zhu D, Shin C, Glassman DT, Abraham N, Watts KL. Patient Satisfaction with Telephone Versus Video-Televisits: A Cross-Sectional Survey of an Urban, Multiethnic Population [published online ahead of print, $2021 \mathrm{Jul} 29$ ]. Urology. 2021;S0090-4295(21)00707-X. https://doi.org/10.1016/j.urology. 2021.05.096

12. Watts KL, Abraham N. "virtually perfect" for some but perhaps not for all: launching telemedicine in the Bronx during the COVID-19 pandemic. J Urol. 2020;204(5):903-4. https://doi.org/ 10.1097/JU.0000000000001185.

Publisher's note Springer Nature remains neutral with regard to jurisdictional claims in published maps and institutional affiliations. 